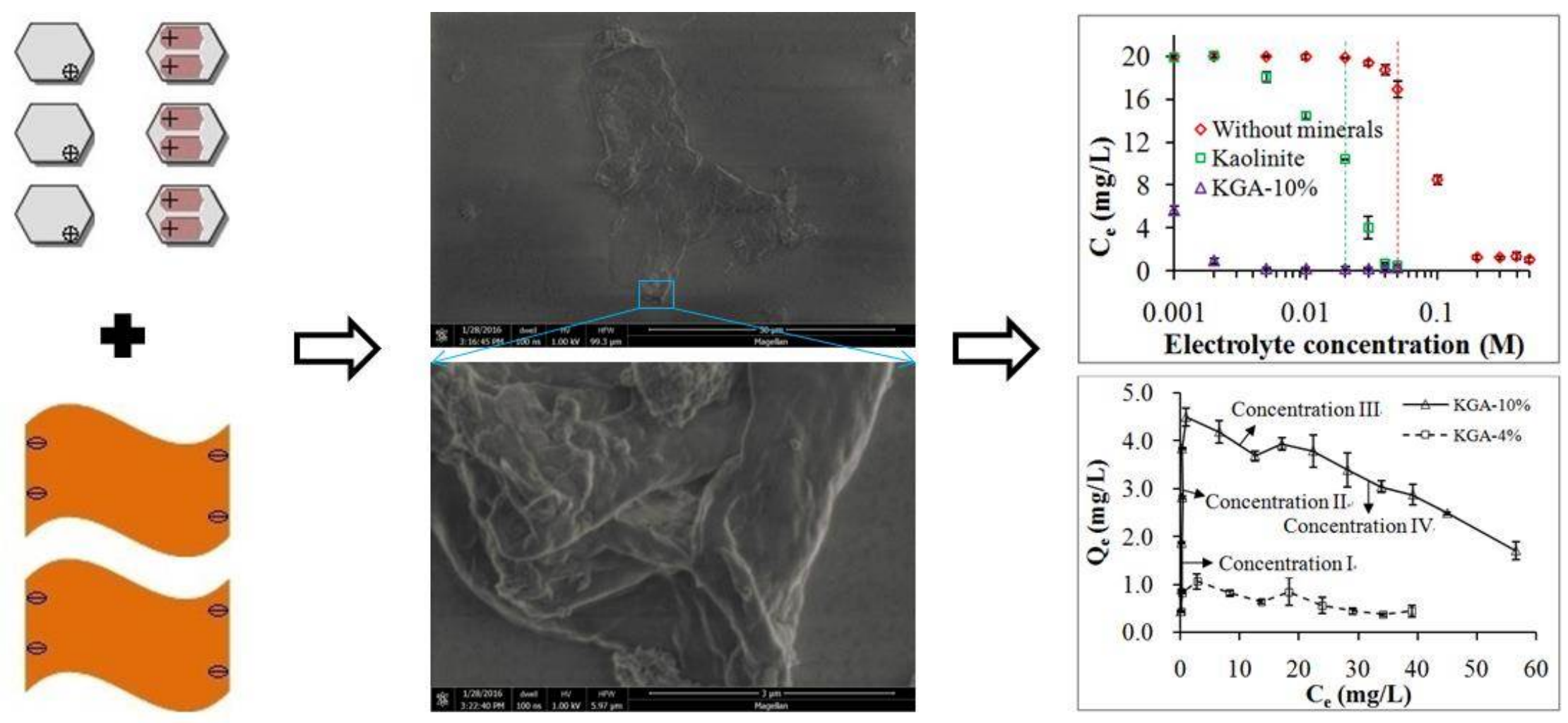

Electrostatic attraction and decrease of energy barrier lead to heteroaggregation of graphene oxide with minerals 


\section{Effect of co-existing kaolinite and goethite on the aggregation of graphene oxide}

\section{2 in the aquatic environment}

3 Guanxing Huang, ${ }^{\dagger, \ddagger}$ Huiyuan Guo, ${ }^{\ddagger}$ Jian Zhao, ${ }^{\S}$ Yonghong Liu, ${ }^{\ddagger}, \neq$ and Baoshan Xing, ${ }^{* \neq}$

$4 \quad{ }^{\dagger}$ Institute of Hydrogeology and Environmental Geology, Chinese Academy of Geological Sciences, Shijiazhuang

5 050061, China

$6 \quad$ † Stockbridge School of Agriculture, University of Massachusetts, Amherst, MA 01003, USA

$7 \quad$ § Institute of Costal Environmental Pollution Control, and Ministry of Education Key Laboratory of Marine

8 Environment and Ecology, Ocean University of China, Qingdao 266100, China

$9 \quad$ \# Department of Chemistry, College of Science, Huazhong Agricultural University, Wuhan 430070, China

10 * Corresponding Author: Phone: +1 413545 5212. E-mail: bx@umass.edu.

11 Abstract:

Broad applications of graphene oxide (GO) will result in the release of GO into aquatic environments, where clay minerals and metal (hydr)oxides are commonly present. Thereby the interactions between GO and a binary system containing clay minerals and metal (hydr)oxides can occur. We investigated the aggregation of GO with kaolinite and kaolinite-goethite associations (KGAs) in aquatic systems under different pHs, ionic strengths, and GO concentrations. GO suspension was unstable at low pHs, and the aggregation of GO occurred in the presence of KGA-4\% and KGA-10\% until pH 5 and 6, respectively. Kaolinite decreased the critical coagulation concentration (CCC) of $\mathrm{GO}$ at $\mathrm{pH} 5.5$ from around 50 to $20 \mathrm{mM} \mathrm{NaCl}$ due to the reduced energy barrier. Heteroaggregation of GO with KGAs was extremely sensitive to ionic strength at $\mathrm{pH} 5.5$, and the CCC of GO in the presence of KGA-10\% increased from less than $1 \mathrm{mM} \mathrm{NaCl}$ to $5 \mathrm{mM} \mathrm{NaCl}$ with the increase of $\mathrm{pH}$ from 5.5 to 9 . The heteroaggregation extent of GO with KGAs was enhanced firstly, then reduced with the increase of GO 
with increasing GO concentrations. These findings are useful for understanding and predicting the fate of GO in the relatively complicated aquatic and soil environments where binary minerals co-exist.

Keywords: Graphene oxide; Kaolinite-goethite associations; Heteroaggregation; Critical coagulation concentration; Ionic strength

\section{Introduction}

Graphene oxide (GO) is a unique two-dimensional carbon nanomaterial with many carboxyl groups on the edge and hydroxyl and epoxy groups on the basal plane. It is usually produced by oxidation of graphite (Chen et al., 2012; Dreyer et al., 2010). Owing to the polar oxygen functional groups, GO is strongly hydrophilic and can be easily dispersed in water (Chowdhury et al., 2013). Nowadays, GO holds great promises in many applications, such as nanomedicine and water purification (Chen and Chen, 2015; Sun et al., 2008; Wang et al., 2013), because its functional groups serve as sites for chemical modification or functionalization (Dreyer et al., 2014). The increasing application and production of GO makes its release into the aquatic environment inevitable during production, transport, usage, and disposal processes (McWilliams, 2012; Zhao et al., 2014).

Although the toxicity of GO to organisms is unclear, some recent studies showed that GO caused cytotoxicity in some bacteria and cells (Combarros et al., 2016; Nguyen et al., 2015), while another recent study reported that purified GO did not have in-vitro cytotoxicity (Ali-Boucetta et al., 2013). Moreover, the environmental impact of GO on some organisms and the fate of inorganic substances can be significant (Mukherjee et al., 2016; Zhao et al., 2014). The environmental impact of GO was highly related to its dispersibility because GO with higher dispersion has more opportunities to directly contact bacterial cells (Das et al., 2013). Therefore, in order to assess the environmental impact of GO, it is essential to investigate the aggregation and fate of GO in the environment.

To date, previous studies have focused on the stability and aggregation kinetics of GO in the aquatic environment over a broad range of solution chemistry conditions (Chowdhury et al., 2013; Wu et al., 2013). The 
results indicated that GO stability was highly dependent on cation valence, ionic strength (IS) and $\mathrm{pH}$, and less affected by the anion valence. Furthermore, the aggregation and deposition of GO in the aquatic environment in the presence of metal oxides have been reported (Ren et al., 2014), indicating that the extent of GO aggregation and deposition in the aquatic environment increased in the presence of metal oxides $\left(\mathrm{Al}_{2} \mathrm{O}_{3}\right)$. In addition, higher interactions of GO with natural organic matter (NOM) coated surfaces than with silica was observed by Chowdhury et al. (2014a). These results facilitate our understanding on the behavior of GO in simple aqueous-solid systems. However, in real environments, natural solid particles are usually associated with each other. How these associations affect the fate of GO in waters is still unknown. Therefore, further investigations are needed to unravel the behavior and fate of GO in the relatively complicated systems.

In our previous study, it is found that goethite could reduce GO dispersion through heteroaggregation while other minerals such as kaolinite cannot influence GO dispersion (Zhao et al., 2015). However, in natural environments, goethite and kaolinite more often associate with each other rather than in discrete, separate form, and the physicochemical properties such as specific surface area and cation exchange capacity of this association are different from that of their individual minerals (Wei et al., 2012; Wei et al., 2014). Therefore, goethite and kaolinite were chosen as the representative hydrous oxides and clay minerals respectively, and the kaolinite-goethite association (KGA) was prepared and used in this study. By using batch experiments, the aggregation of GO with kaolinite alone and KGAs was investigated across a wide range of solution chemistry conditions ( $\mathrm{pH}$ and IS). The findings will provide new insight into the fate of GO in relatively complicated aqueous and porous systems such as aquatic-terrestrial transition zones and aquifers, where hydrous oxides and clay minerals usually co-exist.

\section{Materials and methods}

\subsection{Materials preparation}


GO was prepared by exfoliation of graphite flakes using an improved Hummers' method (Supporting Information (SI), Section 2.1) (Marcano et al., 2010). The dried GO was dispersed and exfoliated in deionized water (DW) under ultrasonication for $3 \mathrm{~h}(20 \mathrm{kHz}$, S-4000, Misonix) to prepare a GO stock suspension (200 $\mathrm{mg} / \mathrm{L}$ ). The $\mathrm{pH}$ and electrical conductivity (EC) of the GO stock suspension were 3.59 and $81.7 \mu \mathrm{S} / \mathrm{cm}$, respectively. Kaolinite was prepared according to the procedure described by Kunze and Dixon with minor modification (SI, Section 2.1) (Kunze and Dixon, 1986). Two KGAs were prepared in this study, which were characterized by about 10\% (KGA-10\%) and 4\% (KGA-4\%) of goethite in the KGAs (equal to 6.3\% and 2.5\% Fe in KGAs), and represented the Fe-rich soils and Fe-poor soils, respectively. KGAs were prepared according to the method described by Wei et al. (2014). Briefly, 200 / 100 mL oxygen-free DW was added to 48.5 / 20.5 g kaolinite in a $1 \mathrm{~L}$ polyethylene flask, followed by vigorous stirring and ultrasonic dispersion. Then 95 / $12 \mathrm{~mL} 1 \mathrm{M} \mathrm{FeCl}_{3}$ solution was poured into the kaolinite suspension under vigorous magnetic stirring, meanwhile the suspension was adjusted to $\mathrm{pH}$ 6. Thereafter pure $\mathrm{N}_{2}$ was flowed through the suspension. After $30 \mathrm{~min}, 5.7$ / $0.72 \mathrm{~mL} 1 \mathrm{M} \mathrm{FeCl}_{2}$ solution was injected into the suspension, while the suspension was adjusted to $\mathrm{pH} 6$ again, and the total volume was adjusted to $1 \mathrm{~L}$. After a reaction time of $30 \mathrm{~min}$, both the stirring and $\mathrm{N}_{2}$ supply were stopped, and the polyethylene flask was tightly sealed and the suspension was aged in a water bath of $55^{\circ} \mathrm{C}$ for $5 \mathrm{~d}$, followed by a rest period of $30 \mathrm{~d}$ at $25^{\circ} \mathrm{C}$. The final product was centrifuged and washed with oxygen-free DW until the conductivity of the supernatant was $<10 \mu \mathrm{S} / \mathrm{cm}$. Then the product was freeze-dried in vacuum $72 \mathrm{~h}$, and ground to pass a 100-mesh sieve (SI, Section 2.1).

\subsection{Batch experiments}

The aggregation experiments were performed in $20-\mathrm{mL}$ vials with Teflon-lined screw caps at $25{ }^{\circ} \mathrm{C}$. In the absence of minerals (unary system), the aggregation of GO as affected by solution chemistry was performed as a function of $\mathrm{pH}(2.0-9.2)$ and IS ( $\mathrm{NaCl}, 0-500 \mathrm{mM})$ with $20 \mathrm{mg} / \mathrm{L}$ of initial GO. For the binary system, $20 \mathrm{mg}$ of 
minerals (kaolinite, KGA-4\%, or KGA-10\%) were added into vials before the addition of solution and GO stock suspension. Correspondingly, the aggregation experiments were performed as a function of the IS ( $\mathrm{NaCl}, 0-50$ $\mathrm{mM})$ at a fixed equilibrium $\mathrm{pH}(5.5 \pm 0.2$ for all minerals, $7.2 \pm 0.2$ for KGA-10\%, $9.0 \pm 0.2$ for KGA-10\%) with $20 \mathrm{mg} / \mathrm{L}$ of initial GO; as a function of GO concentrations (1-60 mg/L) at a fixed equilibrium $\mathrm{pH} 5.0 \pm 0.2$ without electrolyte (only in the presence of KGAs); and as a function of $\mathrm{pH}$ (2.0-9.0) at $20 \mathrm{mg} / \mathrm{L}$ of initial GO without electrolyte. The fixed equilibrium $\mathrm{pH}$ values of the solution in vials were adjusted by adding negligible volumes of 0.01-0.50 M HCl or $\mathrm{NaOH}$. Vials were shaken at a speed of $150 \mathrm{rpm}$ for $24 \mathrm{~h}$ at $25^{\circ} \mathrm{C}$ to reach equilibrium (Figure S1), and then left undisturbed in dark on a flat surface for $24 \mathrm{~h}$ to settle down minerals and GO aggregations (Figure S2). After that, $5 \mathrm{~mL}$ supernatants were removed into 8-mL DW-washed plastic bottles, and the bottles were centrifuged at 3000 rpm for 30 min to separate suspended particles from the supernatants, while the GO in supernatants remained stable after centrifugation (Figures S3 and S4). The GO concentrations in the supernatants $\left(\mathrm{C}_{\mathrm{e}}, \mathrm{mg} / \mathrm{L}\right)$ were determined by UV-vis spectrophotometer (Agilent 8453, USA) at a wavelength of $226 \mathrm{~nm}$ (Figure S5), and the standard curve of GO had a ${ }^{2}$ value of 0.999 (Figure S6).

The release of Fe-related ions in solution from KGA-10\% as a function of $\mathrm{pH}$ was examined. Briefly, $10 \mathrm{~mL}$ of DW was added into vials which already contained $20 \mathrm{mg}$ of KGA-10\%, and the solution $\mathrm{pH}$ was adjusted to a range of 3.5-9.0. After shaking for $24 \mathrm{~h}$, the suspensions were settled for $24 \mathrm{~h}$, and then 5 mL supernatants were centrifuged (3000 rpm, $30 \mathrm{~min})$ and filtered through a Teflon membrane $(0.45 \mu \mathrm{m})$. Concentrations of Fe-related ions in filtrates were determined using UV-vis spectrophotometer (Agilent 8453, USA) at a wavelength of $512 \mathrm{~nm}$ with o-Phenanthroline method (Analytical Methods Committee, 1978). The aggregation experiments at a fixed equilibrium $\mathrm{pH}$ were run in duplicate, while other experiments were run in triplicate. All the experimental data were the averages of duplicate or triplicate determinations.

\subsection{Characterization of GO and minerals}


The electrophoretic mobility (EPM) and hydrodynamic diameter were determined when GO suspension (20

$\mathrm{mg} / \mathrm{L})$ was under a range of $\mathrm{pH}(3.0-9.2)$ and $\mathrm{IS}(\mathrm{NaCl}, 1-500 \mathrm{mM})$ at $25{ }^{\circ} \mathrm{C}$. The zeta potentials of $\mathrm{GO}$ and minerals were measured as a function of $\mathrm{pH}$ and IS at $25{ }^{\circ} \mathrm{C}$. EPM, hydrodynamic diameter, and zeta potential were measured with a particle size analyzer (90Plus, Brookhaven Instruments Co.). To further investigate the morphology and heteroaggregation of GO-KGAs complexes, the GO-KGA-10\% suspensions were dropped onto the surface of silicon wafer disks, and then viewed under scanning electron microscopy (SEM, FEI Magellan 400 XHR) after drying. For comparison, individual KGA-10\% samples were also prepared and observed using SEM integrated with an energy dispersive X-ray spectrometer (EDS, Oxford 80mm² X-Max).

\subsection{Derjaguin-Landau-Verwey-Overbeek (DLVO) interaction energy}

The energy barrier between GO sheets and minerals at different ISs was calculated according to DLVO theory. The total interaction energy between particles $\left(V_{\mathrm{TOT}}\right)$ can be defined as the sum of two interactions, the attractive van der Waals interaction $\left(V_{\mathrm{VDW}}\right)$ and the repulsive electrostatic double layer interaction ( $\left.V_{\mathrm{EDL}}\right)$ (Hogg et al., 1966). Details of the interaction energy calculation were provided in the SI, Section 2.4.

\section{Results and discussion}

\subsection{Influence of pH on GO aggregation}

Aggregation of GO as a function of $\mathrm{pH}(2.0-9.2)$ in the absence and presence of minerals is shown in Figure 1A.

In the absence of minerals, the concentrations of suspended GO in solution decreased dramatically from $\mathrm{pH} 4$ to 2 , indicating that more GO aggregated as a result of weaker electrostatic repulsion between GO sheets caused by the protonation of carboxyl groups located at edges (Shih et al., 2012). Hence, the homoaggregation of GO sheets occurs at low pHs, and probably via the face-to-face pattern considering the large interaction surface area (Wu et al., 2013). By contrast, GO was stable in solution with $\mathrm{pH}$ from 4.7 to 9.2, showing the negligible impact of $\mathrm{pH}$ on the stability of GO in this range. This change of GO concentrations in solution as a function of pH in the absence 
of minerals can be evidenced by the data of EPM and hydrodynamic diameter (Figure S7), supported by the previous studies (Chowdhury et al., 2013; Lanphere et al., 2013; Wu et al., 2013).

A similar tendency of GO stability with the increase of solution $\mathrm{pH}$ was found in the presence of kaolinite. GO suspension was stable when $\mathrm{pH}$ values were higher than 4 (Figure 1A), because little GO-GO homoaggregation occurred according to the above discussion and other previous studies (Chowdhury et al., 2013; Ren et al., 2014). Besides, GO and kaolinite both with negative charges prevent the heteroaggregation of GO with kaolinite due to the electrostatic repulsions (Figure 1B). The zeta potentials results showed that the $\mathrm{pH}_{\mathrm{PzC}}$ of kaolinite without electrolyte was about 3.3 (Figure $1 \mathrm{~B}$ ). Thus, from $\mathrm{pH}_{\mathrm{PZC}}(3.3)$ to $\mathrm{pH} 4$, only the homoaggregation of GO to GO is responsible for GO aggregation in GO_kaolinite binary system, while the heteroaggregation of GO and kaolinite is excluded (Figure 1B). By contrast, as $\mathrm{pH}$ is lower than $\mathrm{pH}_{\mathrm{PZC}}$ (3.3), GO aggregation is not only controlled by the homoaggregation of GO sheets but also affected by the heteroaggregation of GO with kaolinite owing to the electrostatic attractions. For example, the concentrations of GO in solution in GO_kaolinite binary system were lower than that in the absence of kaolinite in the $\mathrm{pH}$ range of 2-3 (Figure 1A). Kaolinite in SEM images showed plate-like shape, and commonly with a lateral size less than $2 \mu \mathrm{m}$ (Figure S8), while the sheet-like shape of GO could be folded (Zhao et al., 2015). Therefore, kaolinite plates were able to be wrapped by GO sheets when heteroaggregation occurred. Moreover, it is known that carboxylic groups $\left(\mathrm{pK}_{\mathrm{a}} 4.3\right)$ on the edge of GO sheets are much earlier to be ionized than hydroxyl groups ( $\mathrm{pK}_{\mathrm{a}}$ 9.8) on the basal plane of GO sheets (Konkena and Vasudevan, 2012). Therefore, heteroaggregates between GO and kaolinite may be formed through kaolinite plates interacting with the edge of GO sheets and then wrapped by $\mathrm{GO}$ at $\mathrm{pH}$ below $\mathrm{pH}_{\mathrm{PzC}}$ (Figure 1C).

GO suspensions became stable in GO-KGA-4\% and GO-KGA-10\% binary systems when pH values were higher than 5 and 6, respectively (Figure 1A). Correspondingly, the $\mathrm{pH}_{\mathrm{PZC}}$ without electrolyte were around 5 and 6 for KGA-4\% and KGA-10\%, respectively (Figure 1B), showing that the extent of GO aggregation as a function 
$\mathrm{pH}$ is controlled by the PZC (above 4) of minerals. GO suspensions were stable in the presence of KGAs as a result of the electrostatic repulsion between $\mathrm{GO}$ and $\mathrm{KGAs}$ when solution $\mathrm{pH}$ was higher than $\mathrm{pH}_{\mathrm{PZC}}$ of $\mathrm{KGAs}_{\text {. On }}$ the contrary, GO aggregated in the presence of KGAs when solution pH was lower than $\mathrm{pH}_{\mathrm{PzC}}$ of KGAs because of the electrostatic attraction between GO and KGAs (Figure 1A). Therefore, heteroaggregation between GO and KGAs is the only aggregation form of GO in GO-KGAs binary systems in the pH range of 4 to PZC, while both the heteroaggregation of GO and KGAs and the homoaggregation of GO sheets are responsible for the GO aggregation in GO-KGAs binary systems below $\mathrm{pH} 4$ (Figure 1C). In addition, below $\mathrm{pH} 4$, the heteroaggregation of $\mathrm{GO}$ and $\mathrm{KGA}-10 \%$ is the dominant form (above $75 \%$ ) of the GO aggregation in GO-KGA-10\% binary system due to less than 25\% GO left in solution around pH 4 (Figure 1A). The goethite formed on the surface of kaolinite was smaller than kaolinite in KGAs (Figure S9), indicating that KGAs plates are also smaller than large GO sheets. Thus, similar to the heteroaggregation between GO and kaolinite, the possible heteroaggregation between GO and KGAs can be proposed that KGAs plates tended to interact with the edge of GO sheets and then wrapped by GO when solution $\mathrm{pH}$ was lower than the $\mathrm{pH}_{\mathrm{PzC}}$ of KGAs (Figure 1C), which can be further evidenced by SEM images of GO-KGAs complexes (Figure S10).

However, these aggregation modes of GO in the presence of KGAs as a function of pH would not be correct if enough Fe-related ions were released from KGAs into the solution, because about or more than $1.008 \mathrm{mg} / \mathrm{L}$ Fe-related ions will significantly destabilize GO in solutions (Zhao et al., 2015). Thus, the release of Fe-related ions from KGA-10\% as a function of $\mathrm{pH}$ was examined in this study with the results showing that the highest concentration of Fe-related ions released from KGA-10\% at the $\mathrm{pH}$ range of 3.5 to 9.0 was only around 0.425 mg/L (Figure S11), thus the impact of dissolved Fe-related ions from KGAs on the aggregation of GO would be negligible.

\subsection{Effect of ionic strength on GO aggregation}


Figure 2A shows the aggregation of $\mathrm{GO}$ as a function of $\mathrm{IS}(\mathrm{NaCl}, 0-500 \mathrm{mM})$ with or without minerals at $\mathrm{pH}$ 5.5. In the absence of minerals, GO suspensions were highly stable and the aggregation was not observed until 20 $\mathrm{mM} \mathrm{NaCl}$. Correspondingly, the hydrodynamic diameter of GO followed a similar trend of GO stability as a function of IS (Figure S12). The critical coagulation concentration (CCC) of $\mathrm{NaCl}$ for $\mathrm{GO}$ aggregation in the absence of minerals was around $50 \mathrm{mM}$, being in agreement with the previous studies (Chowdhury et al., 2013; Zhao et al., 2015). According to the DLVO theory (Chowdhury et al., 2014b; Evans and Wennerström, 1999; Fan et al., 2015; Hua et al., 2015), the aggregation mechanisms are as follows: When IS below the CCC, the decrease of electrical double layer repulsion between GO sheets was improved by the increase of IS (Chen and Elimelech, 2007), which can be evidenced by the increase of EPM and the decrease of energy barrier with IS (Figures S13 and S14A), thus, an increase of IS promoted the aggregation extent of GO (Shih et al., 2012); However, with IS above the CCC, almost all GO charges were screened and the aggregation rate of GO reached a maximum and became independent of the IS, correspondingly, the EPM showed little change with increasing IS, when IS was above $50 \mathrm{mM}$ (Figure S13).

In the GO—kaolinite binary system at $\mathrm{pH} 5.5$, the aggregation of GO was not observed when $\mathrm{NaCl}$ was lower than $2 \mathrm{mM}$. Compared to the unary system, stronger GO aggregation extent was observed in GO-kaolinite binary system with $\mathrm{NaCl}$ concentration from 5 to $50 \mathrm{mM}$ (Figure 2A), indicating that the presence of kaolinite will play an important role in the aggregation of GO in the real aquatic environment where IS can be up to $20 \mathrm{mM}$ (Huang et al., 2013). In addition, the CCC for the aggregation of GO in GO-kaolinite binary system was around $20 \mathrm{mM}$, lower than that in unary system $(50 \mathrm{mM})$. Unlike in the unary system, what is the aggregation mechanism of GO in GO-kaolinite binary system when $\mathrm{NaCl}$ concentration was lower than $20 \mathrm{mM}$ ? On the one hand, according to the above discussion in unary system, the homoaggregation of GO did not occur (pH 5.5) when $\mathrm{NaCl}$ concentration was lower than $20 \mathrm{mM}$ due to the strong electrostatic repulsion between GO sheets (Figure 2B). On 
the other hand, significantly reduced energy barrier of GO was found in the presence of kaolinite at $\mathrm{pH} 5.5$ as a function of IS in comparison with that in the absence of kaolinite (Figure S14B), which indicates that the heteroaggregation between GO and kaolinite would occur in GO_-kaolinite binary system in the relatively low IS (e.g., 5-10 mM). As a result, when $\mathrm{NaCl}$ concentration was lower than $20 \mathrm{mM}$, GO and kaolinite heteroaggregated in this binary system due to the reduced energy barrier of GO by kaolinite. This is also why the CCC in GO_kaolinite binary system was lower than that in unary system. Similarly, previous studies found that clay minerals such as kaolinite and montmorillonite decreased the CCC and the energy barrier of other nanoparticles such as negatively charged Ag nanoparticles under acid condition as a function of IS (Wang et al., 2015; Zhou et al., 2012). In addition, when $\mathrm{NaCl}$ concentration increased from $20 \mathrm{mM}$ to $30 \mathrm{mM}$, the percentage of GO in solution was decreased from about $50 \%$ to $20 \%$ in GO-kaolinite binary system. Meanwhile, a little decrease of GO was also observed in the unary system (Figure 2A), showing that both heteroaggregation between GO and kaolinite and homoaggregation of GO sheets contributed to GO aggregation in GO_kaolinite binary system when $\mathrm{NaCl}$ concentration was higher than $20 \mathrm{mM}$, and heteroaggregation between $\mathrm{GO}$ and kaolinite was the dominant process. It is important to reveal how GO heteroaggregated with kaolinite. At pH 5.5 the basal plane of kaolinite is negatively charged while the edge of kaolinite is positively charged owing to the edge PZC commonly higher than 6 (Tombácz and Szekeres, 2006). It is also known that the basal plane electric double layer (EDL) of clay minerals can spill over the thin edge at low IS, so that the edge EDL is completely masked, but it will be exposed at high IS because the basal plane EDL is compressed with the increase of IS (Tombácz, 2004; Tombácz and Szekeres, 2006). Thus, we proposed a possible heteroaggregation process between GO and kaolinite as follows: when NaCl concentration $>5 \mathrm{mM}$ and $\mathrm{pH}$ at 5.5 , kaolinite plates have negative charges on the basal plane and positive charges on the edge, and the edges of GO carry negative charges (Konkena and Vasudevan, 2012), GO and kaolinite interact with each other via the edges of kaolinite and GO on account of electrostatic attraction, then 
221

222

223

224

225

226

227

228

229

230

231

232

233

234

235

kaolinite plates are wrapped by GO sheets since GO sheets could be folded (Figure S10).

In GO-KGA-10\% binary system at $\mathrm{pH}$ 5.5, at low IS (e.g., below $2 \mathrm{mM}$ ), stronger aggregation extent of GO was observed with the IS (Figure 2A), indicating that the aggregation of GO became more sensitive to the IS in the presence of KGA-10\% compared with GO alone or GO_kaolinite. The CCC in GO_KGA-10\% binary system was less than $1 \mathrm{mM}$, much lower than that in GO_-kaolinite system, thus KGA-10\% had a higher ability than kaolinite to aggregate GO in the aquatic environment. GO heteroaggregated with KGA-10\% in GO_KGA-10\% binary system at $\mathrm{pH}$ 5.5, because KGA-10\% had positive charges while GO had negative charges at $\mathrm{pH} 5.5$ regardless of IS (Figure 2B). Nevertheless, only around $12.5 \%$ GO sheets aggregated with KGA-10\% plates (Here, GO-KGA complex was denoted as "KGA ${ }_{\mathrm{GO}}$ ") in GO-KGA-10\% binary system via electrostatic attraction (Figures S14C and S15) in the absence of electrolytes, and the surface positive charges of KGA-10\% was screened, thereby other $87.5 \%$ GO sheets remained stable in solution due to the electrostatic repulsion between $\mathrm{GO}$ and $\mathrm{KGA}_{\mathrm{GO}}$. When IS increased, even though $\mathrm{KGA}_{\mathrm{GO}}$ had negative charges at $\mathrm{pH} 5.5$ as a function of IS (Table S1), further heteroaggregation between $\mathrm{KGA}_{\mathrm{GO}}$ and GO was still likely to occur with the

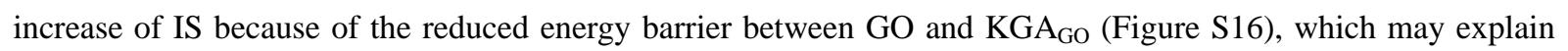
why a sharp decrease of GO concentration was observed as IS increased from $1 \mathrm{mM}$ to 2 mM (Figure $2 \mathrm{~A}$ ). In addition, almost all GO aggregated in GO-KGA-10\% system at $2 \mathrm{mM} \mathrm{NaCl}$, while GO aggregation was not observed in GO alone and GO—-kaolinite system, thus, heteroaggregation of GO with KGA-10\% and KGA $\mathrm{KO}_{\mathrm{G}}$ probably responsible for the GO aggregation in GO-KGA-10\% binary system at pH 5.5 as a function of IS.

In order to further understand the aggregation mechanism of GO in GO-KGA-10\% binary system, the stability of GO suspensions in GO-KGA-10\% binary system as a function of IS was also examined at pH 7.2 and 9 (Figure 3A). When IS was $0 \mathrm{mM}$, unlike at $\mathrm{pH} 5.5$, the aggregation of $\mathrm{GO}$ was not observed in GO-KGA-10\% binary system at pH 7.2 and 9 because of the strong electrostatic repulsion (Figure 1). When IS 
increased, however, aggregation of GO was observed in GO_KGA-10\% binary system at pH 7.2 and 9 (Figure 3A). As discussed above, it should also be attributed to the reduced energy barrier with increasing IS. It is worth mentioning that the CCC of GO in GO-KGA-10\% binary system increased from less than $1 \mathrm{mM}$ to $\sim 5 \mathrm{mM}$ as the $\mathrm{pH}$ values increased from 5.5 to 9 (Figure 3A), due to the increased energy barrier between GO and KGA-10\% with increasing $\mathrm{pH}$, because the reduced zeta potential of KGA-10\% with increasing $\mathrm{pH}$ was found (Figure 3B), and the lower zeta potential means the bigger energy barrier between GO and KGA-10\% at same IS according to the DLVO theory (SI, Section 2.4).

\subsection{Impact of GO concentrations on GO aggregation with KGAs}

Figure 4 shows the aggregation of GO with KGAs at $\mathrm{pH} 5.0$ as a function of GO concentrations without background electrolyte. A critical concentration $\left(\mathrm{C}_{\mathrm{c}}\right)$ for the aggregation of GO and KGA-10\% was observed, and was around the equilibrium GO concentration $\left(\mathrm{C}_{\mathrm{e}}\right)$ of $1.0 \mathrm{mg} / \mathrm{L}$ or the initial GO concentrations $\left(\mathrm{C}_{0}\right)$ of $10 \mathrm{mg} / \mathrm{L}$. When $\mathrm{C}_{\mathrm{e}}$ was lower than $\mathrm{C}_{\mathrm{c}}$, the aggregated amount $\left(\mathrm{Q}_{\mathrm{e}}\right)$ of $\mathrm{GO}$ onto the KGA-10\% increased rapidly, and almost all GO sheets were aggregated with the KGA-10\%. By contrast, When $\mathrm{C}_{e}$ was higher than $\mathrm{C}_{c}$, a significant decrease of $Q_{e}$ was observed with the increase of $C_{e}$. A similar phenomenon was also observed in the aggregation of GO onto KGA-4\%, which also began to decrease as $\mathrm{C}_{\mathrm{e}}$ above $\mathrm{C}_{\mathrm{c}}$. The higher $\mathrm{Q}_{\mathrm{e}}$ in $\mathrm{GO}-\mathrm{KGA}-10 \%$ system than that in GO_KGA-4\% system was attributed to the higher percentage of goethite particles in KGA-10\%.

To investigate the heteroaggregation mechanisms of GO with KGA-10\% at different GO concentrations, SEM was used to examine the aggregation between GO and KGA-10\% at different $\mathrm{C}_{0}$ s. Many KGA plates did not heteroaggregate with $\mathrm{GO}$ at low $\mathrm{C}_{0}$ (i.e., $4 \mathrm{mg} / \mathrm{L}$ ) (Figure 5A), while almost all KGA plates heteroaggregated with GO at relatively higher $\mathrm{C}_{0}$ s (i.e., 10 and $60 \mathrm{mg} / \mathrm{L}$ ) (Figures $5 \mathrm{~B}$ and $5 \mathrm{H}$ ). That is why $\mathrm{Q}_{\mathrm{e}}$ of GO increased rapidly when $\mathrm{C}_{e}$ was lower than $\mathrm{C}_{c}$ (Figures 4 and 6). As it can be seen from SEM images (Figures 5B-5H), we have known that all KGA plates heteroaggregated with different sizes of GO sheets when $\mathrm{C}_{\mathrm{e}}$ was higher than $\mathrm{C}_{\mathrm{c}}$. To 
identify the effect of GO with different sizes on GO-KGA heteroaggregation, a GO stock suspension (around 800 $\mathrm{mg} / \mathrm{L}$ ) filtered with $0.22 \mu \mathrm{m}$ membrane to obtain large-size GO suspension and small-size GO suspension (Figure S17A). It is shown that large-size GO had much more negative charges than that of small-size GO at a same pH (Figure S17B), thus, KGA plates would prefer to aggregate with large-size GO when large-size GO and small-size GO co-exist. Therefore, a possible heteroaggregation scheme is proposed. KGA plates would heteroaggregate with more large-size GO sheets and less small-size GO sheets with the increase of $C_{e}$ when $C_{e}$ was higher than $C_{c}$ (Figure 6), because the amount of large-size GO sheets was increased with the increase of $\mathrm{C}_{\mathrm{e}}$. In this case, goethite particles (positive charge) coated on kaolinite plates would be more efficiently wrapped by GO sheets with the increase of $C_{e}$ (Figure 6). Because many goethite particles would be ineffective when KGA plates were wrapped by large-size GO (Figures 5C-5G), while all goethite particles would be available and effective when KGA plates aggregated with small-size GO (Figures 5B and 5H). As a result, less GO sheets would aggregate with KGA plates with the increase of $C_{e}$ when $C_{e}$ was higher than $C_{c}$ due to KGA plates more efficiently wrapped by GO sheets with the increase of $C_{e}$, and thereby leading to the decrease of $Q_{e}$ with the increase of $C_{e}$ when $C_{e}$ was higher than $\mathrm{C}_{\mathrm{c}}$.

\section{Conclusions and environmental implications}

In natural aqueous environments such as aquatic-terrestrial transition zones, where hydrous oxides and clay minerals usually co-exist and are associated with each other (Wei et al., 2014). The fate of GO in these systems will be not only affected by $\mathrm{pH}$ and IS, but also influenced by its interaction with hydrous oxides and clay minerals. Therefore, to predict the transport and fate of GO in aquatic environments, it is necessary to have a systematic understanding of the aggregation mechanisms in these environments. Our results revealed that GO sheets could be aggregated in the presence of KGA-4\% and KGA-10\% when solution pH was lower than 5 and 6 , respectively. This heteroaggregation between GO and KGA indicates that GO mobility in aquatic environments 
would be highly reduced by the presence of high-PZC minerals (e.g., KGA-10\%). In addition, the aggregation extent of GO and KGA-10\% decreased with the increase of GO concentration when it was higher than a critical concentration (10mg/L, $20 \mathrm{mg} \mathrm{KGA-10 \% ,} 10 \mathrm{~mL}$ GO suspension), indicating that high ratio of GO/KGAs in the aquatic environment could lead to high exposure (possibly toxicity) to aquatic organisms while low ratio of GO/KGAs may cause potential toxicity to benthonic organisms. These findings provide crucial insight regarding the fate and transport of GO in the natural aquatic-terrestrial transition zones and aquifers, where clay minerals and hydrous oxides co-exist. However, it is worth mentioning that mineral surfaces in the environment are often coated with NOM (Kretzschmar and Sticher, 1997), and the aggregation mechanisms of GO with NOM coated minerals is little known. NOM such as humic acid was found to enhance transport of other carbon nanomaterials in saturated porous media (Hyung et al., 2007; Xie et al., 2008). Therefore, further aggregation studies of GO co-affected by minerals and NOM are needed in the future.

\section{Acknowledgments}

This research was supported by USDA-NIFA Hatch program (MAS 00475), Natural Science Foundation of China (41403086), Basic Scientific Study Fund from the Institute of Hydrogeology and Environmental Geology, Chinese Academy of Geological Sciences (SK201611), and the China Geological Survey Grant (12120115048001). Dr. Huang and Dr. Liu thank the China Scholarship Council to support their study at the University of Massachusetts.

\section{References}

Ali-Boucetta, H., Bitounis, D., Raveendran-Nair, R., Servant, A., Van den Bossche, J., Kostarelos, K., 2013. Purified graphene oxide dispersions lack in vitro cytotoxicity and in vivo pathogenicity. Adv Healthc Mater. 2, 433-441.

Analytical Methods Committee, 1978. Standardised general method for the determination of iron with 
Chen, D., Feng, H.B., Li, J.H., 2012. Graphene oxide: preparation, functionalization, and electrochemical applications. Chem. Rev. 112(11), 6027-6053.

Chen, X.X., Chen, B.L., 2015. Macroscopic and Spectroscopic Investigations of the Adsorption of Nitroaromatic Compounds on Graphene Oxide, Reduced Graphene Oxide and Graphene Nanosheets. Environ. Sci. Technol. 49 (10), 6181-6189.

Chen, K.L., Elimelech, M., 2007. Influence of humic acid on the aggregation kinetics of fullerene (C60) nanoparticles in monovalent and divalent electrolyte solutions. J. Colloid Interface Sci. 309 (1), $126-134$.

Chowdhury, I., Duch, M.C., Mansukhani, N.D., Hersam, M.C., 2013. Colloidal properties and stability of graphene oxide nanomaterials in the aquatic environment. Environ. Sci. Technol. 47, 6288-6296.

Chowdhury, I., Duch, M.C., Mansukhani, N.D., Hersam, M.C., Bouchard, D., 2014a. Interactions of Graphene Oxide Nanomaterials with Natural Organic Matter and Metal Oxide Surfaces. Environ. Sci. Technol. 48(16), 9382-9390.

Chowdhury, I., Duch, M.C., Mansukhani, N.D., Hersam, M.C., Bouchard, D., 2014b. Deposition and release of graphene oxide nanomaterials using a quartz crystal microbalance. Environ. Sci. Technol. 48, 961-969.

Combarros, R.G., Collado, S., Díaz, M., 2016. Toxicity of graphene oxide on growth and metabolism of Pseudomonas putida. J. Hazard. Mater. 310, 246-252.

Das, S., Singh, S., Singh, V., Joung, D., Dowding, J.M., Reid, D., Anderson, J., Zhai, L., Khondaker, S.I., Self, W.T., Seal, S., 2013. Oxygenated functional group density on graphene oxide: Its effect on cell toxicity. Part. Part. Syst. Charact. 30, 148-157.

Dreyer, D.R., Park, S.J., Bielawski, C.W., Ruoff, R.S., 2010. The chemistry of graphene oxide. Chem. Soc. Rev. 39 (1), 228-240. 
Dreyer, D.R., Todd, A.D., Bielawski, C.W., 2014. Harnessing the chemistry of graphene oxide. Chem. Soc. Rev. 43, 5288-5301.

Evans, D.F., Wennerström, H., 1999. The Colloidal Domain: Where Physics, Chemistry, Biology, and Technology Meet (2nd Edition); Wiley-VCH: New York.

Fan, W., Jiang, X., Yang, W., Geng, Z., Huo, M., Liu, Z., Zhou, H., 2015. Transport of graphene oxide in saturated porous media: Effect of cation composition in mixed Na-Ca electrolyte systems. Sci. Total Environ. 511, 509-515.

Hogg, R., Healy, T.W., Fuerstenau, D.W., 1966. Mutual coagulation of colloidal dispersions. Trans. Faraday Soc. 62, 1638.

Huang, G., Sun, J., Zhang, Y., Chen, Z., Liu, F., 2013. Impact of anthropogenic and natural processes on the evolution of groundwater chemistry in a rapidly urbanized coastal area, South China. Sci. Total Environ. 463-464, 209-221.

Hua, Z., Tang, Z., Bai, X., Zhang, J., Yu, L., Cheng, H., 2015. Aggregation and resuspension of graphene oxide in simulated natural surface aquatic environments. Environ. Pollut. 205, 161-169.

Hyung, H., Fortner, J.D., Hughes, J.B., Kim, J.H., 2007. Natural organic matter stabilizes carbon nanotubes in the aqueous phase. Environ. Sci. Technol. 41 (1), 179-184.

Konkena, B., Vasudevan, S., 2012. Understanding aqueous dispersibility of graphene oxide and reduced graphene oxide through $\mathrm{pK}_{\mathrm{a}}$ measurements. J. Phys. Chem. Lett. 3, 867-872.

Kretzschmar, R., Sticher, H., 1997. Transport of humic-coated iron oxide colloids in a sandy soil: Influence of $\mathrm{Ca}^{2+}$ and trace metals. Environ. Sci. Technol. 31, 3497-3504.

Kunze, G.W., Dixon, J.B., 1986. Pretreatment for mineralogical analysis, In: Klute, A. (Ed.), Methods of Soil Analysis: Part 1. Physical and Mineralogical Methods, 2nd ed. ASA, Madison, WI. 
Lanphere, J.D., Luth, C.J., Walker, S.L., 2013. Effects of solution chemistry on the transport of graphene oxide in saturated porous media. Environ. Sci. Technol. 47 (9), 4255-4261.

Marcano, D.C., Kosynkin, D.V., Berlin, J.M., Sinitskii, A., Sun, Z., Slesarev, A., Alemany, L.B., Lu, W., Tour, J.M., 2010. Improved synthesis of graphene oxide. ACS Nano 4, 4806-4814.

McWilliams, A., 2012. Graphene: Technologies, applications, and markets. BCC Research, AVM075B, http://www.bccresearch.com/market-research/advanced-materials/graphene-technologies-applications-marketsavm075b.html

Mukherjee, R., Bhunia, P., De, S., 2016. Impact of graphene oxide on removal of heavy metals using mixed matrix membrane. Chem. Eng. J. 292, 284-297.

Nguyen, T.H.D., Lin, M., Mustapha, A., 2015. Toxicity of graphene oxide on intestinal bacteria and caco-2 cells. J. Food Protect. 78 (5), 996-1002.

Ren, X., Li, J., Tan, X., Shi, W., Chen, C., Shao, D., Wen, T., Wang, L., Zhao, G., Sheng, G., Wang, X., 2014. Impact of $\mathrm{Al}_{2} \mathrm{O}_{3}$ on the aggregation and deposition of graphene oxide. Environ. Sci. Technol. 48 (10), 5493-5500.

Shih, C.J., Lin, S.C., Sharma, R., Strano, M.S., Blankschtein, D., 2012. Understanding the pH-dependent behavior of graphene oxide aqueous solutions: A comparative experimental and molecular dynamics simulation study. Langmuir 28 (1), 235-241.

Sun, X., Liu, Z., Welsher, K., Robinson, J.T., Goodwin, A., Zaric, S., Dai, H., 2008. Nano-graphene oxide for cellular imaging and drug delivery. Nano Res. 1, 203-212.

Tombácz, E., Szekeres, M., 2006. Surface charge heterogeneity of kaolinite in aqueous suspension in comparison with montmorillonite. Appl. Clay Sci. 34 (1-4), 105-124.

Tombácz, E., 2004. Colloidal behavior of aqueous montmorillonite suspensions: The specific role of $\mathrm{pH}$ in the 
presence of indi erent electrolytes. Appl. Clay Sci. 27, 75-94.

Wang, C., Li, H., Liao, S., Zheng, H., Wang, Z., Pan, B., Xing, B., 2013. Coadsorption, desorption hysteresis and sorption thermodynamics of sulfamethoxazole and carbamazepine on graphene oxide and graphite. Carbon 65, 243-251.

Wang, H.T., Dong, Y.N., Zhu, M., Li, X., Keller, A.A., Wang, T., Li, F.T., 2015. Heteroaggregation of engineered nanoparticles and kaolin clays in aqueous environments. Water Res. 80, 130-138.

Wei, S.Y., Tan, W.F., Liu, F., Zhao, W., Weng, L.P., 2014. Surface properties and phosphate adsorption of binary systems containing goethite and kaolinite. Geoderma 213, 478-484.

Wei, S.Y., Tan, W.F., Zhao, W., Yu, Y.T., Liu, F., Koopal, L.K., 2012. Microstructure, interaction mechanisms and stability of binary systems containing goethite and kaolinite. Soil Sci. Soc. Am. J. 76 (2), 389-398.

Wu, L., Liu, L., Gao, B., Muñoz-Carpena, R., Zhang, M., Chen, H., Zhou, Z.H., Wang, H., 2013. Aggregation kinetics of graphene oxides in aqueous solutions: Experiments, mechanisms, and modeling. Langmuir 29 (49), $15174-15181$.

Xie, B., Xu, Z.H., Guo, W.H., Li, Q.L., 2008. Impact of natural organic matter on the physicochemical properties of aqueous C60 nanoparticles. Environ. Sci. Technol. 42 (8), 2853-2859.

Zhao, J., Wang, Z., White, J., Xing, B., 2014. Graphene in the aquatic environment: Adsorption, dispersion, toxicity and transformation. Environ. Sci. Technol. 48, 9995-10009.

Zhao, J., Liu, F.F., Wang, Z.Y., Cao, X.S., Xing, B.S., 2015. Heteroaggregation of Graphene Oxide with Minerals in Aqueous Phase. Environ. Sci. Technol. 49, 2849-2857.

Zhou, D.X., Abdel-Fattah, A.I., Keller, A.A., 2012. Clay particles destabilize engineered nanoparticles in aqueous environments. Environ. Sci. Technol. 46 (14), 7520-7526. 


\section{$1 \quad$ Figures}

2

3
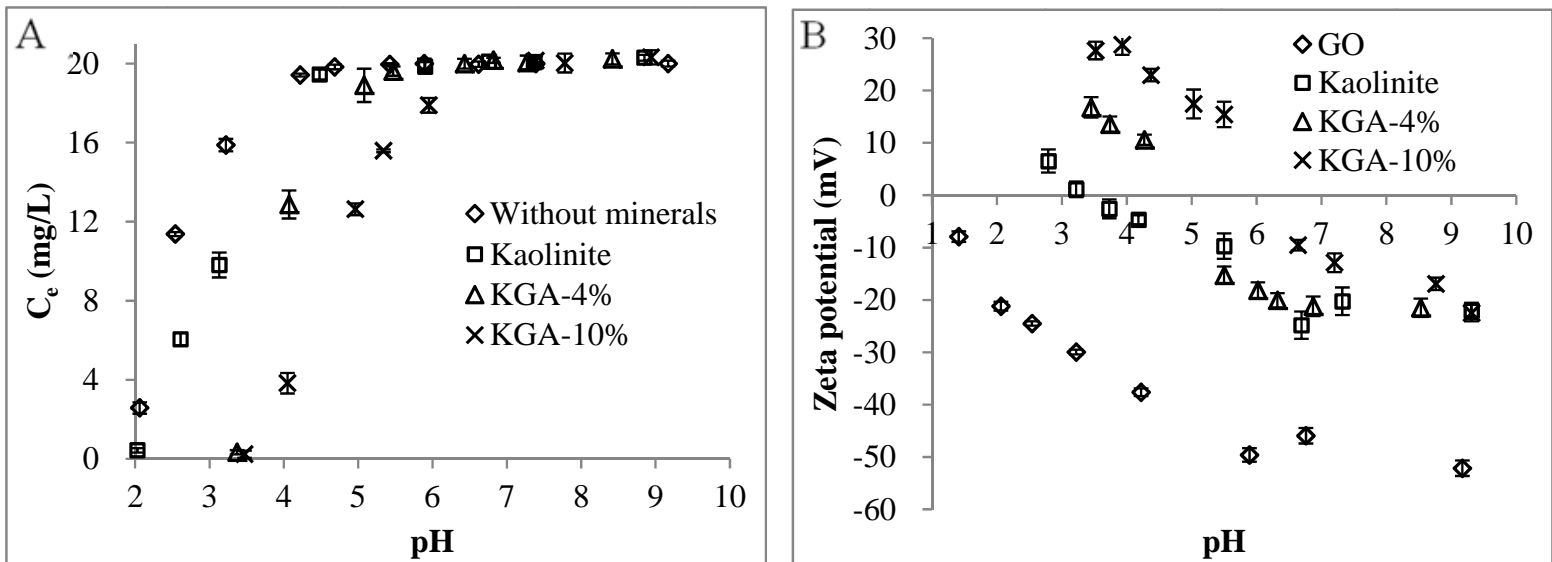

C In the presence of kaolinite

In the presence of $\mathrm{KGA}-4 \%$
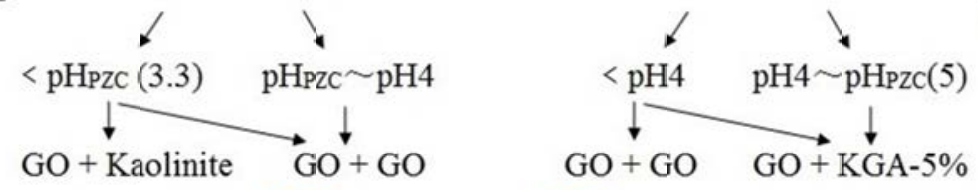

In the presence of $\mathrm{KGA}-10 \%$
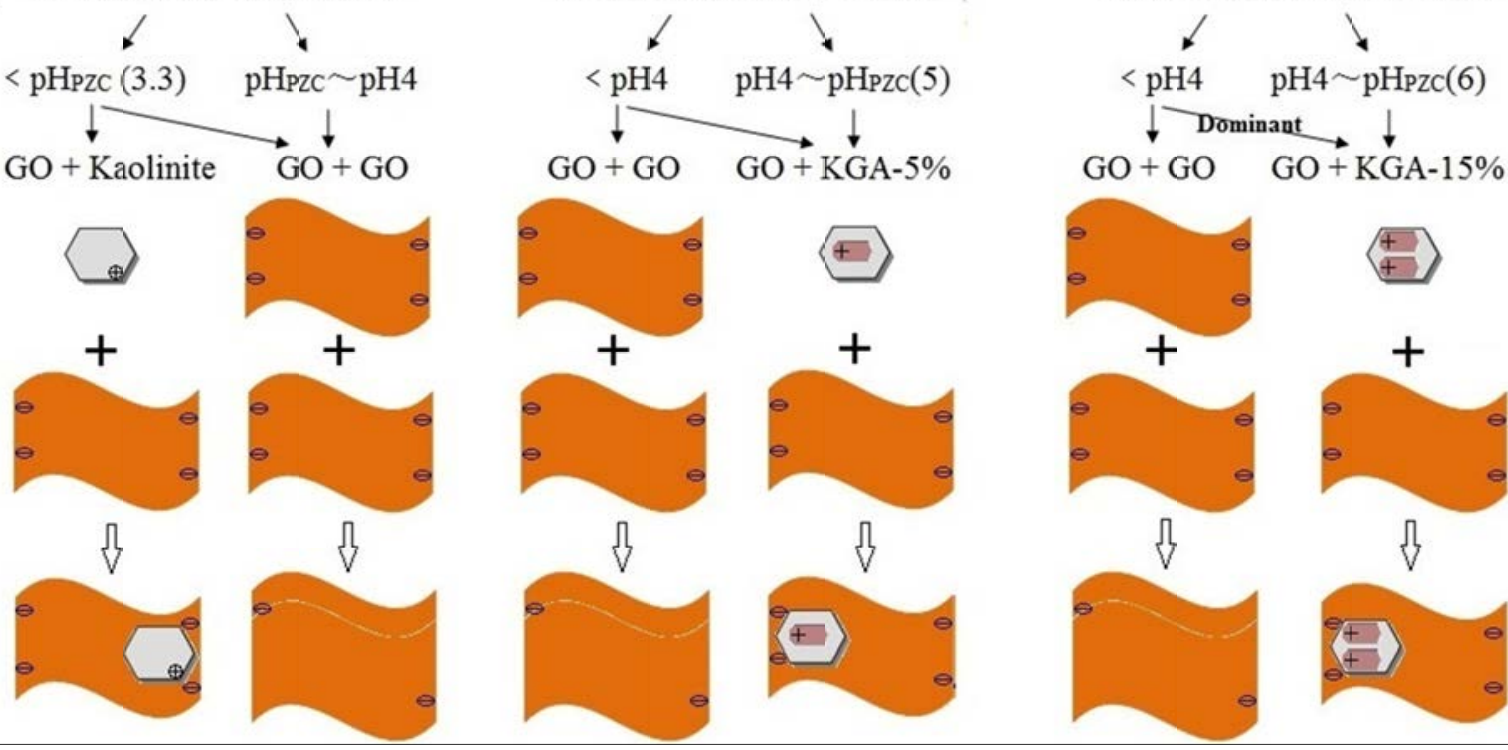

Figure 1. (A) Concentrations of GO in the supernatant as a function of $\mathrm{pH}$ in the absence and presence of minerals

6 with $20 \mathrm{mg} / \mathrm{L}$ of initial GO (without background electrolyte). (B) Zeta potentials of GO and minerals as a function

7 of $\mathrm{pH}(\mathrm{GO}$ concentration $=20 \mathrm{mg} / \mathrm{L}$, minerals $=2 \mathrm{~g} / \mathrm{L}$, without background electrolyte). (C) Illustrative diagram of aggregation modes of GO in the presence of minerals as a function of $\mathrm{pH}$. 

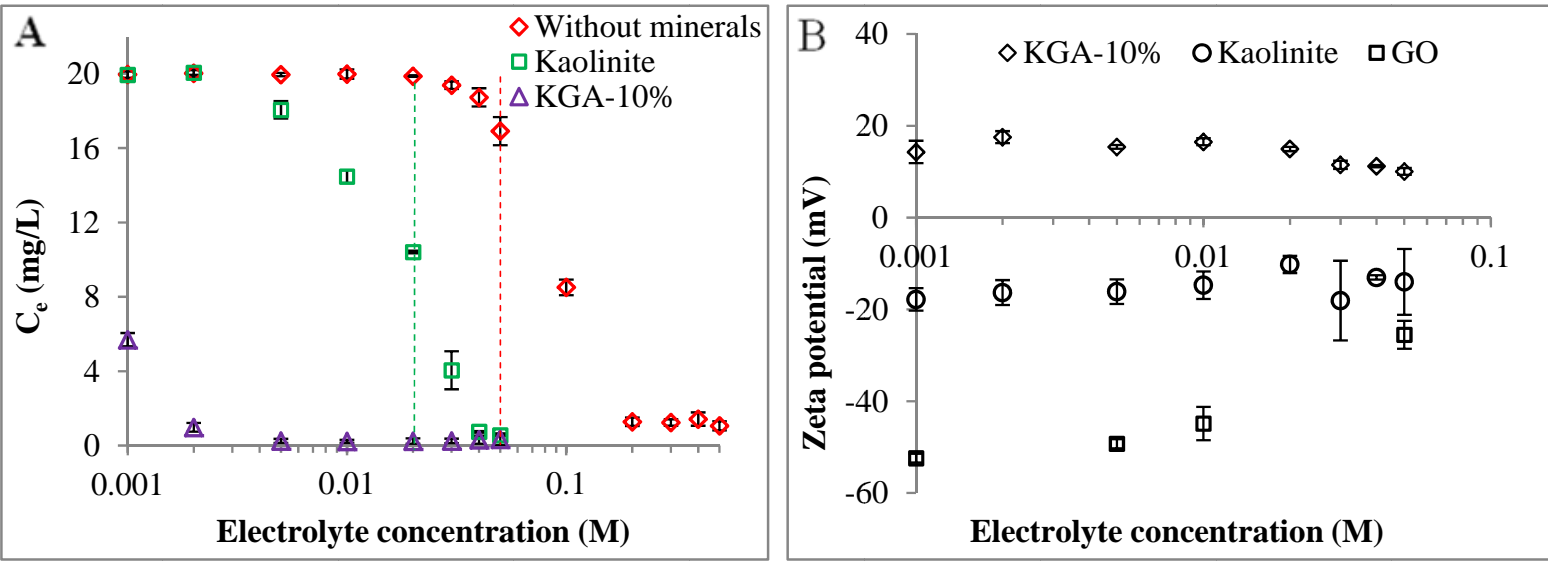

Figure 2. (A) Concentrations of GO in the supernatant as a function of IS in the absence and presence of minerals

with $20 \mathrm{mg} / \mathrm{L}$ of initial $\mathrm{GO}$ at $\mathrm{pH}$ 5.5. (B) Zeta potentials of $\mathrm{GO}$ and minerals as a function of IS at $\mathrm{pH} 5.5$ (GO concentration $=20 \mathrm{mg} / \mathrm{L}$, minerals $=2 \mathrm{~g} / \mathrm{L})$.

13

14

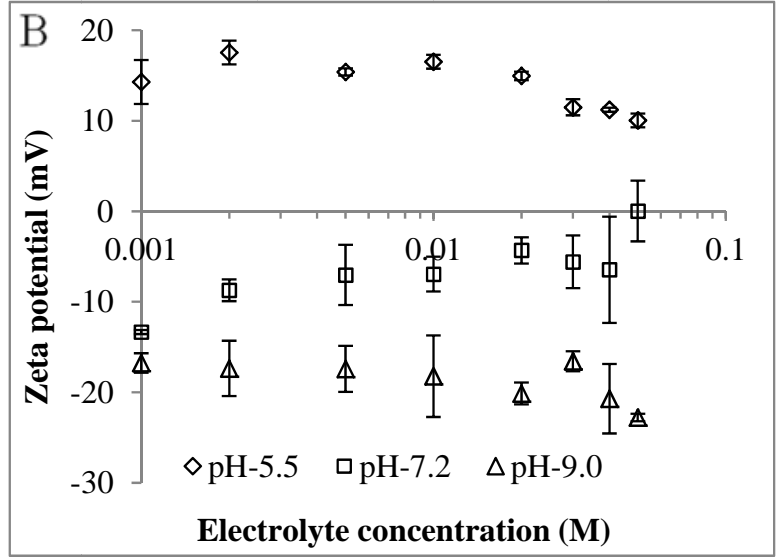

Figure 3. (A) Concentrations of GO in the supernatant as a function of IS in the presence of KGA-10\% with 20 mg/L of initial GO at different pH. (B) Zeta potentials of KGA-10\% as a function of IS at different pH. 


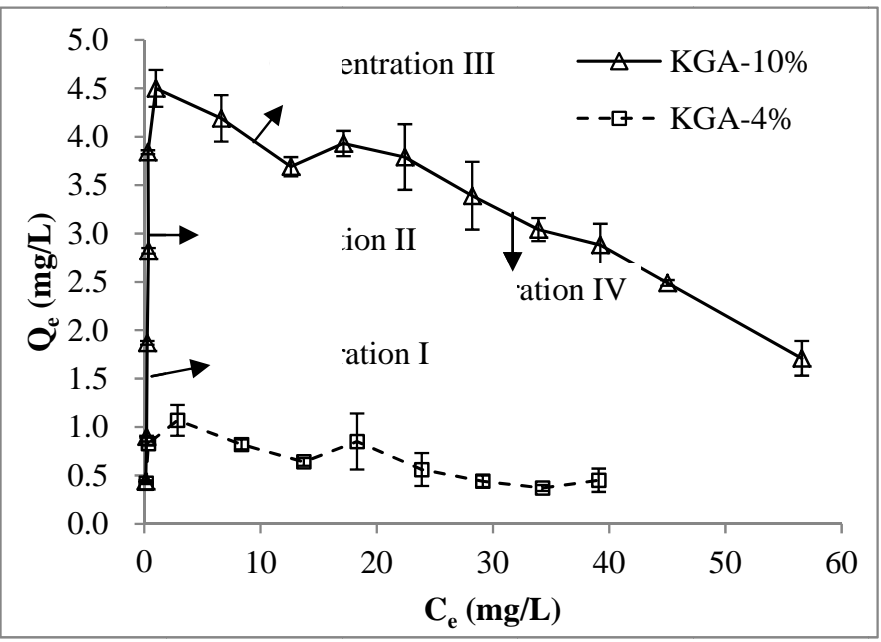

Figure 4. Aggregation of GO with KGAs at $\mathrm{pH} 5.0$ as a function of GO concentrations without background

0.5-1 $\mathrm{C}_{\mathrm{c}}, \mathrm{C}_{0}=1-3 \mathrm{C}_{\mathrm{c}}$, and $\mathrm{C}_{0}=3-6 \mathrm{C}_{\mathrm{c}}$, respectively).
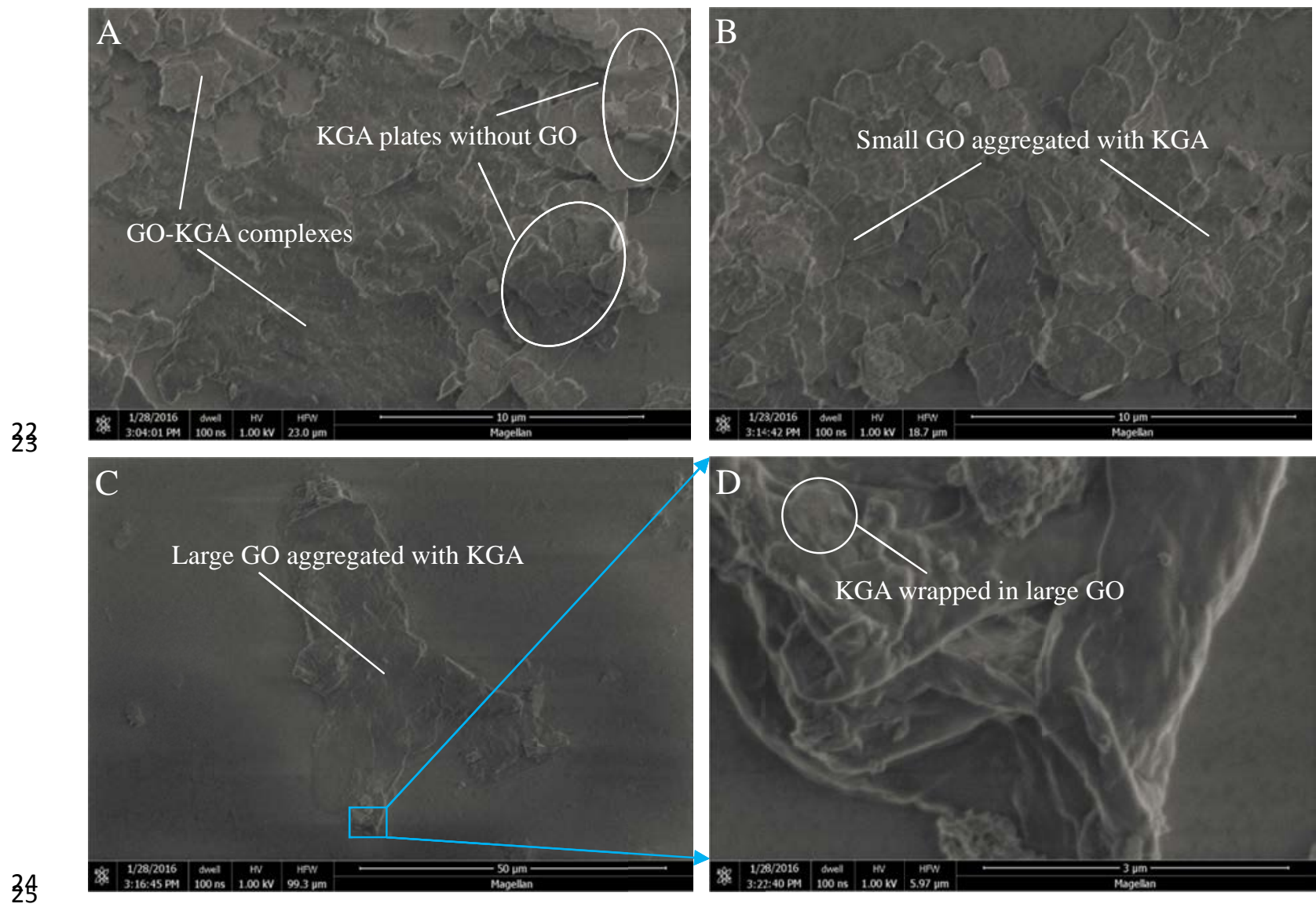


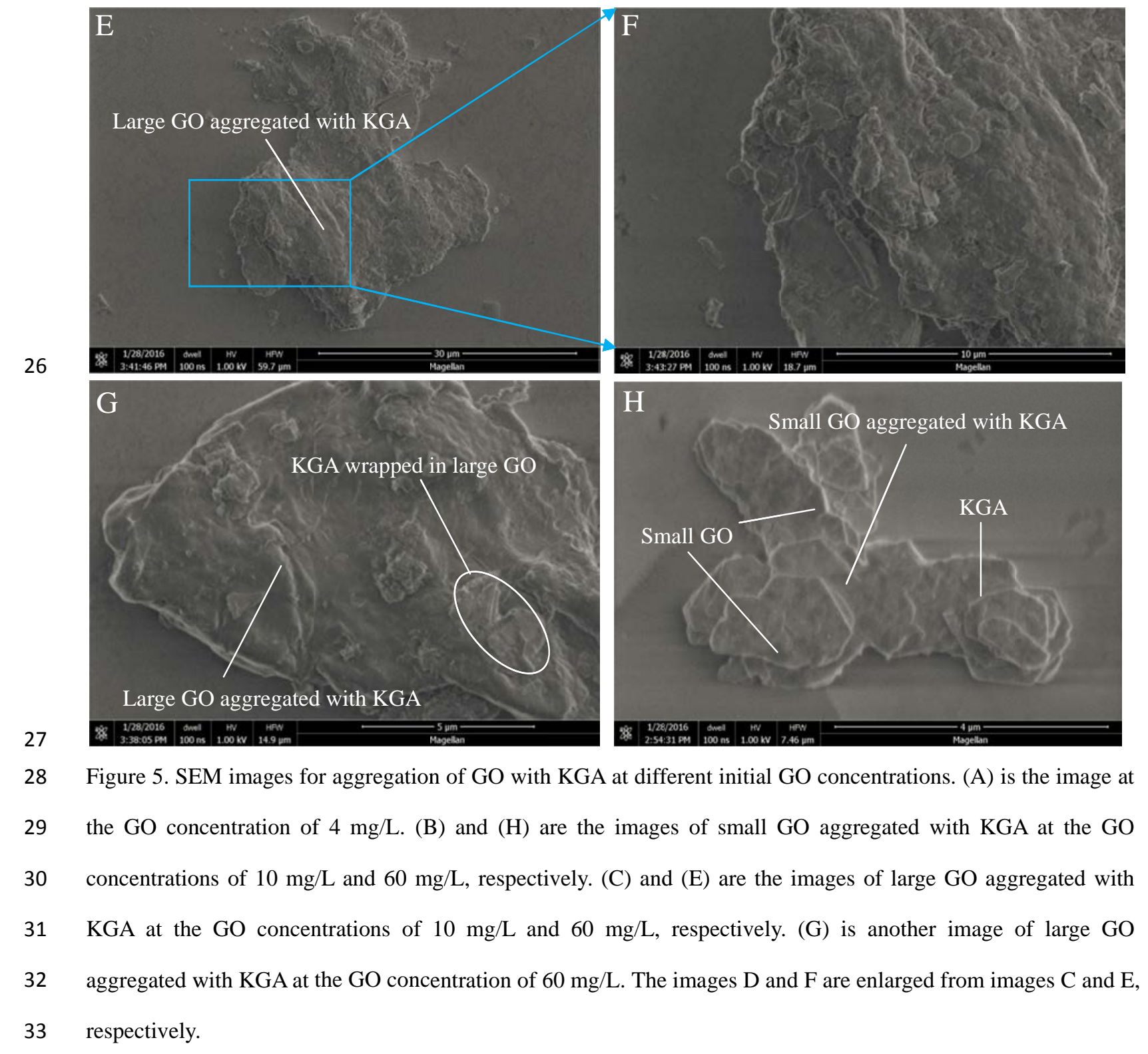




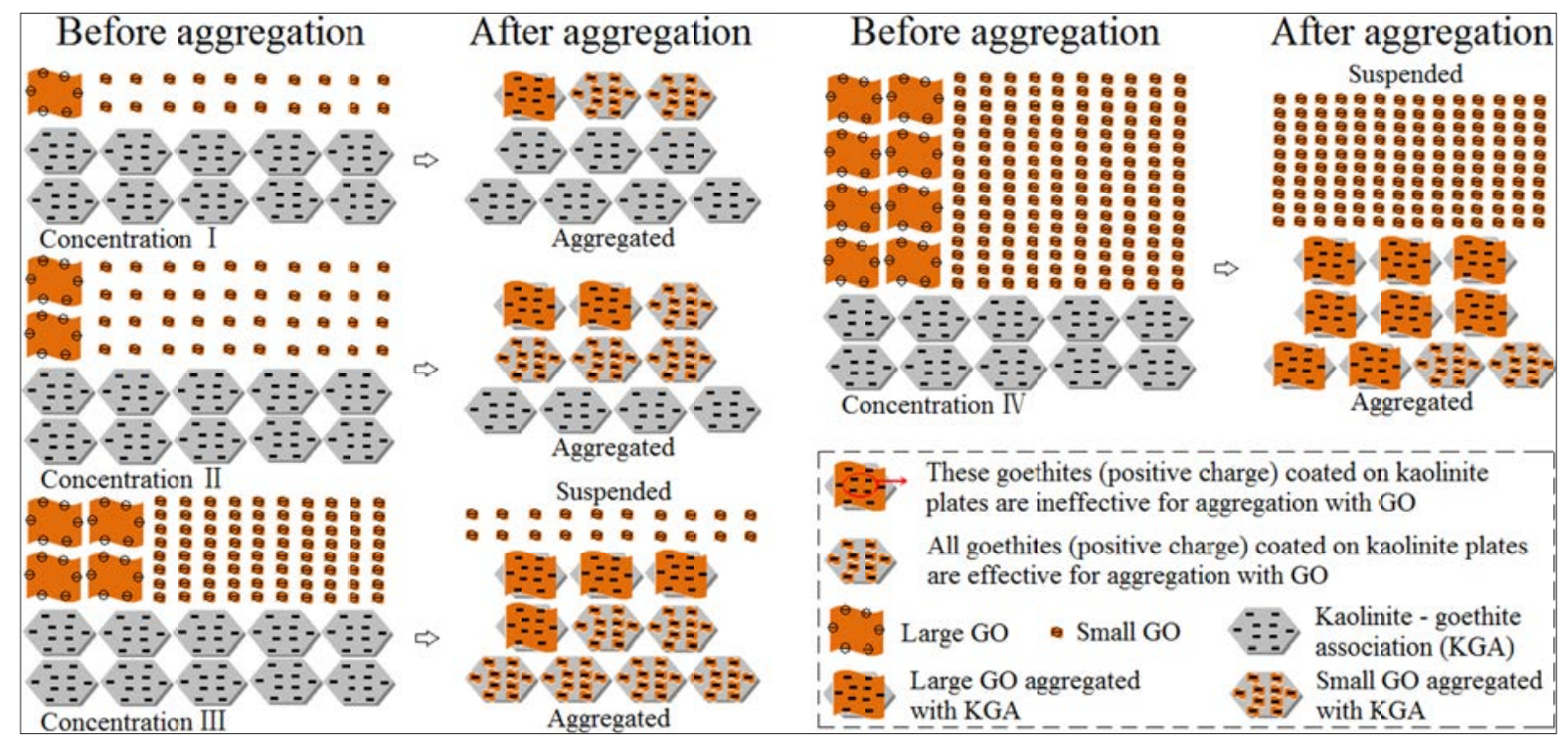

Figure 6. The possible schematic diagram for the aggregation of GO sheets and KGA plates at different GO

concentrations. In the schematic diagram, concentrations I, II, III, IV represent $\mathrm{C}_{0}<0.5 \mathrm{C}_{\mathrm{c}}, \mathrm{C}_{0}=0.5-1 \mathrm{C}_{\mathrm{c}}, \mathrm{C}_{0}=$

1-3 $C_{c}$, and $C_{0}=3-6 C_{c}$, respectively. Less than half KGA plates aggregated with $G O$ at concentration I; more than half KGA plates aggregated with GO at concentration II; all KGA plates aggregated with GO at concentrations III and IV, and the number of KGA plates aggregated with small-size GO at concentration IV is less than that at concentration III, thus leaving more GO sheets in solution instead of on the KGA. 